\title{
openheart Increased baseline ECG R-R dispersion predicts improvement in systolic function after atrial fibrillation ablation
}

\author{
Ryan J Koene, ${ }^{\oplus 1}$ Eric Buch, ${ }^{2}$ Young-Ji Seo, ${ }^{3}$ Jian-Ming Li, ${ }^{4}$ Mackenzi Mbai, ${ }^{4}$ \\ Y Chandrashekhar, ${ }^{4}$ Kalyanam Shivkumar, ${ }^{2}$ Venkatakrishna N Tholakanahalli ${ }^{5}$
}

\begin{abstract}
- Additional material is published online only. To view please visit the journal online (http://dx.doi.org/10.1136/ openhrt-2018-000958).
\end{abstract}

To cite: Koene RJ, Buch E, Seo Y-J, et al. Increased baseline ECG R-R dispersion predicts improvement in systolic function after atrial fibrillation ablation. Open Heart 2019;6:e000958. doi:10.1136/ openhrt-2018-000958

Received 30 0ctober 2018 Revised 14 April 2019 Accepted 30 May 2019
Check for updates

(c) Author(s) (or their employer(s)) 2019. Re-use permitted under CC BY-NC. No commercial re-use. See rights and permissions. Published by BMJ.

For numbered affiliations see end of article.

Correspondence to Dr Venkatakrishna N Tholakanahalli; thola001@ umn.edu

\section{ABSTRACT}

Background Atrial fibrillation (AF) is associated with left ventricular (LV) systolic dysfunction which may improve after AF ablation. We hypothesised that increased ventricular irregularity, as measured by $\mathrm{R}-\mathrm{R}$ dispersion on the baseline ECG, would predict improvement in the left ventricular ejection fraction (LVEF) after AF ablation. Methods Patients with LVEF $<50 \%$ at two US centres (2007-2016), having both a preablation and postablation echocardiogram or cardiac MRI, were included. LVEF improvement was defined as absolute increase in LVEF by $>7.5 \%$. Multivariable logistic regression (restricted to echocardiographic/ECG variables) was performed to evaluate predictors of LVEF improvement.

Results Fifty-two patients were included in this study. LVEF improved in 30 patients (58\%) and was unchanged/ worsened in 22 patients (42\%). Those with versus without LVEF improvement had an increased baseline $\mathrm{R}-\mathrm{R}$ dispersion $(645 \pm 155 \mathrm{~ms}$ vs $537 \pm 154 \mathrm{~ms}, \mathrm{p}=0.02$, respectively). The average baseline heart rate in all patients was 93 beats per minute. After multivariable logistic regression, increased $\mathrm{R}-\mathrm{R}$ dispersion (OR $1.59,95 \% \mathrm{Cl} 1.00$ to $2.55, \mathrm{p}=0.03$ ) predicted LVEF improvement.

Conclusions Increased R-R dispersion on ECG was independently associated with improved systolic function after AF ablation. This broadens the existing knowledge of arrhythmia-induced cardiomyopathy, demonstrating that irregular electrical activation (as measured by increased R-R dispersion on ECG) is associated with a cardiomyopathy capable of improving after AF ablation.

\section{INTRODUCTION}

Arrhythmia-induced cardiomyopathy (AIC) is defined as left ventricular (LV) systolic dysfunction occurring as a result of a persistent cardiac arrhythmia. Although classically attributed to fast heart rates in tachyarrhythmias, frequent premature ventricular contractions (PVCs), which are usually not associated with tachycardia, ${ }^{1}$ as well as persistent atrial fibrillation (AF), in the absence of tachycardia (ie, rate-controlled $\mathrm{AF}$ ), ${ }^{2}$ can also be associated with LV systolic dysfunction. This raises the possibility that, apart from traditional explanations like loss of

\section{Key questions}

What is already known about this subject?

- Catheter ablation is associated with improvement in left ventricular (LV) ejection fraction in certain patients with cardiomyopathy.

What does this study add?

- To the best of our knowledge, this is the first study in humans demonstrating that increased ventricular irregularity, which was assessed by the R-R dispersion on ECG, is associated with improvement in LV ejection fraction after atrial fibrillation ablation.

How might this impact on clinical practice?

- The readily available measurement of ECG R-R dispersion may help to identify those patients most likely to derive LV systolic function improvement after catheter ablation.

atrioventricular synchrony and atrial contraction (ie, the 'atrial kick'), or associated valvular regurgitation, the irregular sequence of ventricular cycle lengths itself can lead to haemodynamic impairment in AF. This could then trigger reduced cardiac output and subsequent neurohormonal activation that can result in LV systolic dysfunction. ${ }^{23}$

It is often unclear whether $\mathrm{AF}$ is the result of an underlying cardiomyopathy (primary cardiomyopathy) or an actual cause of the cardiomyopathy (primary arrhythmia). Some clues that may be suggestive of a primary AIC include rapid AF ventricular response or young, otherwise healthy patients with no other apparent underlying cause for cardiac disease. Additionally, tachycardia-induced cardiomyopathy (TIC) is associated with relatively smaller LV dimensions compared with those with idiopathic dilated cardiomyopathy. ${ }^{4}$ However, severe LV enlargement does not exclude the possibility of TIC, and $\mathrm{AF}$ and heart failure often coexist in patients with multiple comorbidities, several of which are shared risk factors (eg, hypertension, diabetes, ischaemic heart disease and valvular 
heart disease) for both AF and heart failure. ${ }^{5}$ Therefore, the distinction between a primary AIC due to AF and a primary cardiomyopathy causing AF becomes increasingly challenging. Identifying those with a primary AIC has important implications, as an aggressive rate and rhythm control strategy in these patients would presumably result in improvement in their cardiomyopathy.

We hypothesised that greater R-R interval dispersion might be a marker for an arrhythmia-induced cardiomyopathy and therefore also identify patients who would recover systolic function post-AF ablation. ${ }^{6}$

\section{METHODS \\ Study patients}

We reviewed the Minneapolis VA Health Care System and University of California, Los Angeles (UCLA) AF ablation databases (2007-2016), including patients with complete transthoracic echocardiographic (TTE) or cardiac MRI studies before (within 1 year) and after ( $>5$ weeks) AF ablation. The latter was chosen to allow time for reverse remodelling on restoration of sinus rhythm following $\mathrm{AF}$ ablation. Patients with an LVEF $\geq 50 \%$ were excluded. All patients underwent pulmonary vein isolation using radiofrequency ablation with or without left atrial ablation for complex fractionated atrial electrograms.

\section{Echocardiographic and cardiac MRI measurements of LV variables}

The two-dimensional (2D) echocardiographic studies, including chamber quantification measurements, were performed by experienced cardiac sonographers and interpreted and verified by cardiologists certified in 2D echocardiography. Variables of LV function that were measured included LV internal diameter during end-diastole (LVIDd), LV internal diameter during end-systole (LVIDs), LVEF and left atrial diameter. Measurements were performed according to the American Society of Echocardiography chamber quantification guidelines. ${ }^{7}$ All cardiac MRI studies were performed on Siemens Prisma 3T or Siemens Avanto 1.5T MRI scanners (Siemens Healthineers, Malvern, Pennsylvania, USA), with horizontal long axis steady-state free precession images of the right and left ventricles reviewed on a dedicated GE-PACS workstation. Images were reviewed by a cardiovascular radiologist with 10 years of experience. Measurements were performed three times with the mean selected and recorded.

\section{ECG measurements}

$\mathrm{R}-\mathrm{R}$ dispersion was measured as the difference between the longest and shortest R-R interval on the single, most recent ECG preceding AF ablation that demonstrated AF. This was then corrected for a heart rate of 70 beats per minute (bpm) using the formula for corrected R-R dispersion which is (heart rate/70 bpm)*R-R dispersion. The longest or shortest R-R interval could involve a PVC if present.

\section{Definitions}

Improvement in LVEF was defined as $>7.5 \%$ absolute increase in LVEF in comparison to the pre-AF ablation imaging study. A $>7.5 \%$ cut-off was used given the constraint of previously reported $\sim 7.5 \%$ interobserver variability in the measurement of $2 \mathrm{D}$ ejection fraction (EF) ${ }^{8}$ Consequently, LVEF that improved by $\leq 7.5 \%$ compared with the pre-AF ablation imaging study was categorised as unchanged or worsened.

\section{Statistical analysis}

Continuous and categorical variables were summarised as mean \pm SD and frequency (\%), respectively. Patients were categorised into two groups according to whether or not LVEF improved by $>7.5 \%$. The groups were compared using the Pearson's $\chi^{2}$ test for categorical variables and the Student's t-test for continuous variables. Changes in pre-AF versus post-AF ablation echocardiographic or cardiac MRI parameters were compared using the paired t-test. All $\mathrm{p}$ values were two sided with significance of $<0.05$. Univariable analysis was performed on collected clinical data and stratified by patients with and without LVEF improvement. Multivariable logistic regression (restricted to only echocardiographic and ECG variables with $\mathrm{p}<0.10$ due to the small sample size) was performed to calculate ORs of LVEF improvement. Model performance was analysed using the area under the receiver operating curve (C-statistic). Statistical analyses were performed using JMP version 14.1.0 (SAS Institute, Cary, North Carolina, USA).

This study was approved by the Institutional Review Board at the Minneapolis VA Health Care System and UCLA.

\section{RESULTS}

\section{Study population}

Between 1 January 2007 and 31 December 2015, a total of 69 out of 252 consecutive patients undergoing AF ablation at the Minneapolis VA Medical Center had LV dysfunction (LVEF $<50 \%)$ on a preablation echocardiogram (TTE) within 1 year. Of these, a total of 42 had postablation imaging studies and were therefore included in this analysis. We included an additional 10 patients from the UCLA AF ablation database (2012 through 2017) that had both preablation and postablation cardiac MRI $(n=8)$ or echocardiographic data $(n=2)$. The total sample size was 52 patients. All were men.

Baseline demographic and clinical characteristics in the study population as a whole and in the subgroups of patients with improved and not improved LVEF after $\mathrm{AF}$ ablation are presented in table 1 . The mean age of the study group was $63 \pm 8$ years and all patients were men. Sixty per cent of patients had either persistent or long-standing persistent $\mathrm{AF}$, and the remaining had paroxysmal AF. Nearly half $(42 \%)$ had ischaemic heart disease. The mean heart rate was $93 \mathrm{bpm}$, which is satisfactory according to current guidelines, making 
Arrhythmias and sudden death

Table 1 Baseline characteristics in the study population as a whole, stratified by postablation response in LVEF ( $>7.5 \%)$

\begin{tabular}{|c|c|c|c|c|}
\hline Variables & All patients, $n=52$ & LVEF improved, $n=30$ & $\begin{array}{l}\text { LVEF } \\
\text { not improved, } n=22\end{array}$ & P value* \\
\hline Age (years) & $63 \pm 8$ & $63 \pm 8$ & $62 \pm 7$ & 0.83 \\
\hline Male (\%) & 100 & 100 & 100 & 1.00 \\
\hline $\operatorname{LVEF}(\%)$ & $36 \pm 8$ & $36 \pm 8$ & $36 \pm 9$ & 0.70 \\
\hline Hypertension (\%) & 83 & 73 & 95 & 0.04 \\
\hline Diabetes mellitus (\%) & 12 & 13 & 9 & 0.64 \\
\hline COPD (\%) & 21 & 20 & 23 & 0.81 \\
\hline OSA $(\%)$ & 35 & 23 & 50 & 0.05 \\
\hline Prior Ml (\%) & 15 & 10 & 23 & 0.21 \\
\hline Ischaemic heart disease (\%) & 42 & 37 & 50 & 0.34 \\
\hline $\mathrm{CHA}_{2} \mathrm{DS}_{2}$ VASc score & $2.6 \pm 1$ & $2.4 \pm 1$ & $3.0 \pm 1$ & 0.13 \\
\hline CRT or ICD $(\%)$ & 12 & 7 & 18 & 0.24 \\
\hline Body mass index $\left(\mathrm{kg} / \mathrm{m}^{2}\right)$ & $31 \pm 6$ & $29 \pm 5$ & $32 \pm 7$ & 0.08 \\
\hline Persistent/long-standing AF (\%) & 60 & 57 & 64 & 0.62 \\
\hline \multicolumn{5}{|l|}{ Medications } \\
\hline Beta blocker (\%) & 85 & 83 & 86 & 0.76 \\
\hline ACEi/ARB (\%) & 67 & 60 & 77 & 0.19 \\
\hline Spironolactone (\%) & 17 & 13 & 23 & 0.38 \\
\hline Hydralazine and ISDN (\%) & 2 & 0 & 5 & 0.24 \\
\hline Digoxin $(\%)$ & 29 & 30 & 27 & 0.83 \\
\hline Diuretics (\%) & 37 & 33 & 41 & 0.58 \\
\hline Antiarrhythmic (\%) & 44 & 40 & 50 & 0.47 \\
\hline \multicolumn{5}{|l|}{ ECG/echocardiographic } \\
\hline Heart rate (bpm) & $93 \pm 20$ & $97 \pm 22$ & $89 \pm 16$ & 0.15 \\
\hline Corrected R-R dispersion (ms) & $600 \pm 162$ & $645 \pm 155$ & $537 \pm 154$ & 0.02 \\
\hline LVIDd (mm) & $58 \pm 8$ & $54 \pm 6$ & $58 \pm 8$ & 0.03 \\
\hline LVIDs (mm) & $44 \pm 8$ & $42 \pm 7$ & $46 \pm 9$ & 0.06 \\
\hline $\operatorname{LAD}(\mathrm{mm})$ & $49 \pm 9$ & $48 \pm 9$ & $50 \pm 8$ & 0.18 \\
\hline Valvular (\%) & 12 & 7 & 18 & 0.20 \\
\hline
\end{tabular}

*Improved ( $\triangle \mathrm{LVEF}>7.5 \%)$ versus not improved.

ACEi, ACE inhibitor; AF, atrial fibrillation; ARB, angiotensin receptor blocker;COPD, chronic obstructive pulmonary disease;CRT,

Cardiac Resynchronization Therapy; ICD, implantable cardioverter defibrillator; ISDN, isosorbide dinitrate; LVEF, left ventricular ejection fraction;LVIDd, left ventricular internal diameter during end-diastole; LVIDs, left ventricular internal diameter during end-systole; MI, myocardial infarction; OSA, obstructive sleep apnoea; bpm, beats per minute.

tachycardia-mediated cardiomyopathy less likely. A substantial proportion of patients had multiple comorbidities (table 1). Preablation medications included beta blockers in $85 \%$ of patients, an ACE inhibitors or an angiotensin II receptor blockers (ACEi/ARB) in 67\%, antiarrhythmic agents in $44 \%$, diuretics in $37 \%$, digoxin in $29 \%$, as well as spironolactone and the combination of hydralazine with isosorbide dinitrate combination in a minority of patients (table 1 ). The mean LVEF was $36 \% \pm 8 \%$.

\section{Periprocedural changes in cardiac chamber dimensions}

Cardiac chamber measurements before and after AF ablation in the study population as a whole, and also stratified by subsequent EF improvement, are presented in table 2 .
The mean LVEF significantly improved following AF ablation in the whole study cohort as a whole $(36 \% \pm 8 \%$ vs $47 \% \pm 13 \%$, $\mathrm{p}=0.002$ ). For the whole group, there was also a statistically significant reduction in LVIDs (from $44 \pm 8$ $\mathrm{mm}$ to $40 \pm 9 \mathrm{~mm}, \mathrm{p}=0.02$ ). There were no statistically significant changes in other cardiac chamber measurements (ie, left atrial diameter or LVIDd) with AF ablation (table 2).

In the subgroups stratified by LVEF improvement versus no LVEF improvement, there was a statistically significant reduction in LVIDs in the group with LVEF improvement before versus after AF ablation (from $42 \pm 7$ $\mathrm{mm}$ to $36 \pm 6 \mathrm{~mm}, \mathrm{p}=0.001$ ). In contrast, no significant change in LVIDs was seen in the group with unchanged 
Table 2 Left ventricular measurements before and after AF ablation in the study population as a whole, stratified by post-AF ablation response in LVEF (>7.5\%)

\begin{tabular}{|c|c|c|c|c|c|c|c|c|c|}
\hline \multirow[b]{2}{*}{ Variables } & \multicolumn{3}{|c|}{ All patients, $n=52$} & \multicolumn{3}{|c|}{ LVEF improved, $\mathrm{n}=30$} & \multicolumn{3}{|c|}{ LVEF not improved, $n=22$} \\
\hline & Before & After & $P$ value & Before & After & $P$ value & Before & After & $P$ value \\
\hline LVEF, \% & $36 \pm 8$ & $47 \pm 13$ & 0.002 & $36 \pm 8$ & $56 \pm 8$ & $<0.001$ & $36 \pm 9$ & $36 \pm 9$ & 0.74 \\
\hline LVIDs, mm & $44 \pm 8$ & $40 \pm 9$ & 0.02 & $42 \pm 7$ & $36 \pm 6$ & 0.001 & $46 \pm 9$ & $45 \pm 8$ & 0.74 \\
\hline $\mathrm{LAD}, \mathrm{mm}$ & $49 \pm 9$ & $48 \pm 7$ & 0.39 & $48 \pm 9$ & $47 \pm 6$ & 0.38 & $50 \pm 8$ & $49 \pm 8$ & 0.78 \\
\hline
\end{tabular}

Values presented as mean \pm SD.

AF, atrial fibrillation; LAD, left atrial diameter; LVEF, left ventricular ejection fraction; LVIDd, left ventricular internal diameter during enddiastole; LVIDs, left ventricular internal diameter during end-systole.

or worsened LVEF. There were no other periprocedural changes in cardiac measurements (table 2).

\section{Predictors of LVEF improvement after AF ablation}

After a median (IQR) of 5.7 months (4-12 months), LVEF improved by $>7.5 \%$ in 30 patients $58 \%$ of the study population) and was unchanged/worsened in 22 patients (42\%). Pre-AF and post-AF ablation LVEF was $36 \% \pm 8 \%$ vs $56 \% \pm 8 \%$, respectively $(\mathrm{p}<0.001)$, in patients with improved LVEF and $36 \% \pm 9 \%$ vs $36 \% \pm 9 \%$, respectively $(\mathrm{p}=0.74)$, in patients with unchanged/worsened LVEF (table 2).

Patients with improved LVEF after ablation were less likely to have hypertension or sleep apnoea (table 1). Those with versus without LVEF improvement had increased R-R dispersion ( $645 \pm 155 \mathrm{~ms}$ vs $537 \pm 154 \mathrm{~ms}$, respectively, $\mathrm{p}=0.02$ ) (table 1 , figure 1 ) and smaller LVIDd ( $54 \pm 6 \mathrm{~mm}$ vs $58 \pm 8 \mathrm{~mm}, \mathrm{p}=0.02$ ) (table 3 , figure 2 ). Other cardiac chamber dimensions were not associated with changes in LVEF. Multivariable analysis, restricted to echocardiographic and ECG variables (due to limitations in the sample size of the study) having a univariable $\mathrm{p}<0.10$ was performed. The candidate predictors satisfying $\mathrm{p}<0.05$ after multivariable logistic regression included R-R dispersion by ECG (table 4), having an OR (95\% CI) of 1.59 (1.00 to 2.55) ( $\mathrm{p}=0.03)$ for LVEF improvement by $7.5 \%$.

In a subgroup analysis of ischaemic (42\%) versus non-ischaemic heart disease (58\%), LVEF improvement

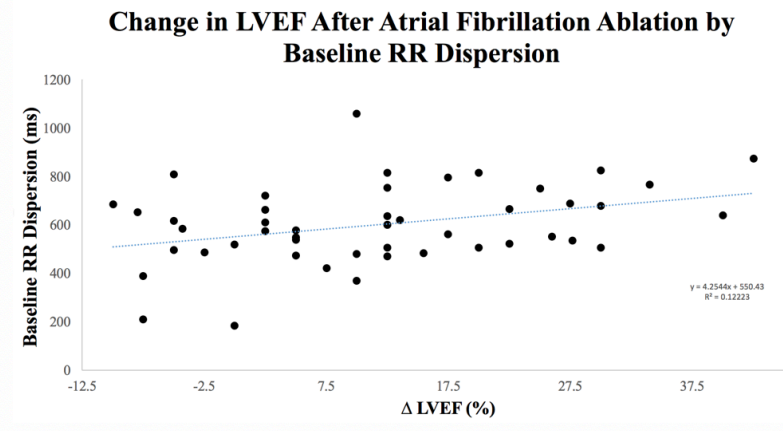

Figure 1 Change in left ventricular ejection fraction (LVEF) after atrial fibrillation ablation based on the baseline R-R dispersion. occurred in $50 \%$ vs $63 \%$, respectively $(\mathrm{p}=0.29)$. In this subgroup analysis, increased R-R dispersion remained a significant predictor of LVEF improvement in those without ischaemic heart disease $(\mathrm{p}<0.01)$, whereas statistical significance was lost in those with ischaemic heart disease (online supplementary table 1).

Baseline R-R dispersion between patients with obstructive sleep apnoea (OSA) $(628 \pm 194 \mathrm{~ms})$ versus those without OSA $(587 \pm 146 \mathrm{~ms})$ was not statistically significant $(\mathrm{p}=0.43)$.

\section{AF recurrence and symptom improvement at 1 year}

At the 1-year follow-up period, $33(66 \%)$ patients had symptom improvement (table 3). Among the cardiac chamber variables, an improvement in symptoms versus unchanged or worsened symptoms at 1 year had smaller left atrial diameter dimensions $(47 \pm 7 \mathrm{~mm}$ vs $52 \pm 7 \mathrm{~mm}$, $\mathrm{p}=0.03$, respectively) (table 3 ). Symptoms improved in $82 \%$ of those with EF improvement, versus only $45 \%$ of those without EF improvement $(\mathrm{p}=0.001)$ (data not shown).

At the 1-year follow-up period, 26 patients (50\%) had no AF recurrences. Among the cardiac chamber variables,

Table 3 Baseline chamber dimensions stratified by future outcomes

\begin{tabular}{llll}
\hline Outcome & LVIDd & LVIDs & LAD \\
\hline Improved LVEF $(\mathrm{n}=30)$ & $54 \pm 6$ & $42 \pm 7$ & $48 \pm 9$ \\
Worsened/unchanged LVEF $(\mathrm{n}=22)$ & $58 \pm 8$ & $46 \pm 9$ & $50 \pm 8$ \\
P value & 0.02 & 0.07 & 0.53 \\
No AF recurrence $(\mathrm{n}=26)$ & $56 \pm 7$ & $44 \pm 7$ & $47 \pm 7$ \\
AF recurrence & $56 \pm 8$ & $43 \pm 9$ & $51 \pm 10$ \\
P value & 0.70 & 0.67 & 0.14 \\
Symptoms improved $(\mathrm{n}=33)$ & $55 \pm 6$ & $43 \pm 7$ & $47 \pm 7$ \\
Symptoms not improved $(\mathrm{n}=17)$ & $58 \pm 8$ & $45 \pm 7$ & $52 \pm 7$ \\
P value & 0.14 & 0.44 & 0.03 \\
\hline
\end{tabular}

All measurements are expressed in millimetres.

$A F$, atrial fibrillation; LAD, left atrial diameter; LVEF, left ventricular ejection fraction; LVIDd, left ventricular internal diameter during end-diastole; LVIDs, left ventricular internal diameter during endsystole. 


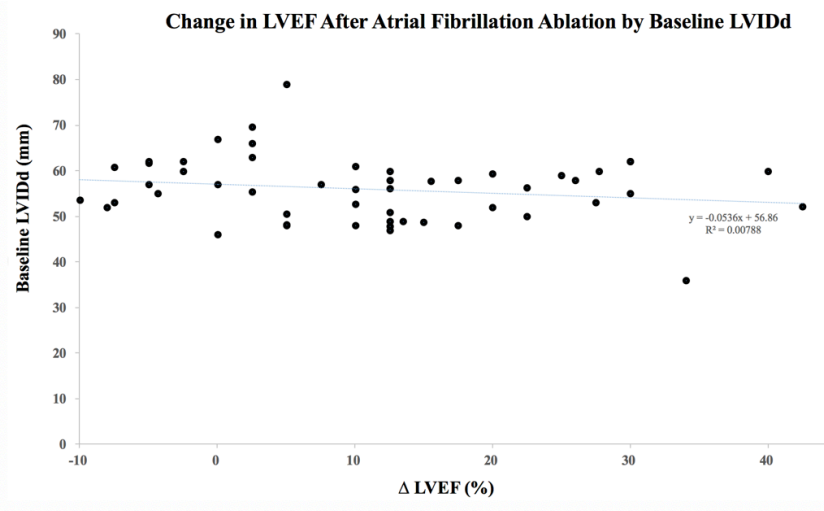

Figure 2 Change in LVEF after atrial fibrillation ablation based on baseline left ventricular internal dimension in diastole. LVEF, left ventricular ejection fraction; LVIDd, left ventricular internal diameter during end-diastole.

there was a trend toward AF recurrence being associated with larger left atrial dimensions $(\mathrm{p}=0.14)($ table 4$)$.

\section{DISCUSSION}

In this study, we found that increased ventricular irregularity, as measured by the R-R dispersion on the most recent ECG demonstrating AF preceding catheter ablation, predicted LVEF improvement following AF ablation. Because AF ablation eliminates or reduces ventricular irregularity (by reducing AF burden), we can surmise that ventricular irregularity itself is a contributing factor to LV systolic dysfunction. In addition, smaller LVIDd on baseline cardiac imaging identified an increased likelihood for LVEF improvement. There was also a trend toward smaller baseline LVIDs and improved postablation $\operatorname{LVEF}(\mathrm{p}=0.06)$, suggesting that better baseline contractility might be an important predictor of postablation response. After multivariable analysis, increased R-R dispersion was the only statistically significant predictor of LVEF improvement among ECG and echocardiographic variables. Perhaps, the principal finding of this

Table 4 Multivariable OR and 95\% Cls for left ventricular EF improvement by $7.5 \%$ after catheter ablation for atrial fibrillation*

EF improvement by $7.5 \%(n=30)$

\begin{tabular}{lll}
\hline $\begin{array}{l}\text { Variables } \\
\begin{array}{l}\text { R-R dispersion (per } 100 \text { unit } \\
\text { increase) }\end{array}\end{array}$ & OR $(95 \%$ value \\
$\begin{array}{l}\text { LVIDd, } \mathrm{mm} \text { (per } 10 \text { unit } \\
\text { increase) }\end{array}$ & $0.57(0.10$ to 3.17$)$ & $0.55)$ \\
$\begin{array}{l}\text { LVIDs, mm (per } 10 \text { unit } \\
\text { increase) }\end{array}$ & $0.78(0.18$ to 3.37$)$ & 0.74 \\
\hline
\end{tabular}

*Restricted to echocardiographic and ECG variables only due to limited sample size, keeping all variables from table 1 with univariable $p$ value $<0.10$.

$E F$, ejection fraction; LVIDd, left ventricular internal diameter during end-diastole; LVIDs, left ventricular internal diameter during endsystole. study is that the R-R dispersion was measured using only a single ECG before AF ablation and may serve as a practical prediction guide. Similarly, LV dimensions which are readily available in most patients undergoing AF ablation can serve to inform postablation outcomes. These findings may have important implications in the management of patients with coexisting $\mathrm{AF}$ and heart failure by helping to identify those who may derive the most benefit from restoration of sinus rhythm with catheter ablation.

Mechanisms for AIC in rate-controlled AF have been postulated but are difficult to study in humans, who often have comorbidities or concomitant tachycardia. ${ }^{3}$ It is speculated that irregular ventricular cycles result in reduced LV filling during short cycles that is incompletely compensated for during long cycles, causing reduced cardiac output and subsequent neurohormonal activation, leading to adverse cardiac remodelling. ${ }^{2}$ A prior pacing study in a canine model showed that straight pacing (ie, regularly paced ventricular cycles) alone reduced cardiac output, but the addition of irregular ventricular cycles further reduced cardiac output by an additional $7 \%{ }^{8}$ Using isolated cardiac myocytes from rats, Ling et al found that irregular pacing compared with regular pacing over a 24-hour period resulted in a substantial decrease in sarcoplasmic reticulum $\mathrm{Ca}^{2+}$-ATPase and phosphorylated phospholamban, both of which are associated with heart failure (figure 3 ).${ }^{9} \mathrm{In}$ LV samples obtained from patients with end-stage heart failure with either persistent $\mathrm{AF}$ or sinus rhythm, there was a decrease in several calcium-handling proteins observed in those with persistent AF but not in those who had sinus rhythm. ${ }^{9}$

Studies have demonstrated improvement in LVEF after catheter ablation in well rate-controlled patients. In a study of 58 consecutive patients with an LVEF $<45 \%$ undergoing catheter ablation for $\mathrm{AF}$, patients improved their LVEF by $17 \% \pm 15 \%$ despite having adequate rate control defined as a heart rate $<80 \mathrm{bpm}$ on 48 hours telemetry preprocedure, and not surprisingly, those with poorly controlled heart rates had even greater improvement of their LVEF by $23 \% \pm 10 \% .{ }^{10}$ A recent randomised controlled trial of 68 patients with adequate heart rate control demonstrated an improvement in LVEF of $18 \% \pm 13 \%$ in patients assigned to catheter ablation versus an LVEF change of $4.4 \% \pm 13 \%$ in those assigned to a rate control strategy. ${ }^{11}$

The findings from our study and these other studies suggest that arrhythmia itself is a source of cardiomyopathy in AF independent of tachycardia. Eliminating the severity of arrhythmia, by way of reducing the R-R dispersion, is associated with improved LV systolic function. Because of this, one might hypothesise that patients with $\mathrm{AF}$, heart failure and increased R-R dispersion should derive the most benefit from a catheter-based rhythm control therapeutic strategy.

Subject to the limitations of the study, these observations add to our understanding of the pathophysiology of AIC. This study may also help identify patients more likely to have an arrhythmia-induced or arrhythmia-worsening 


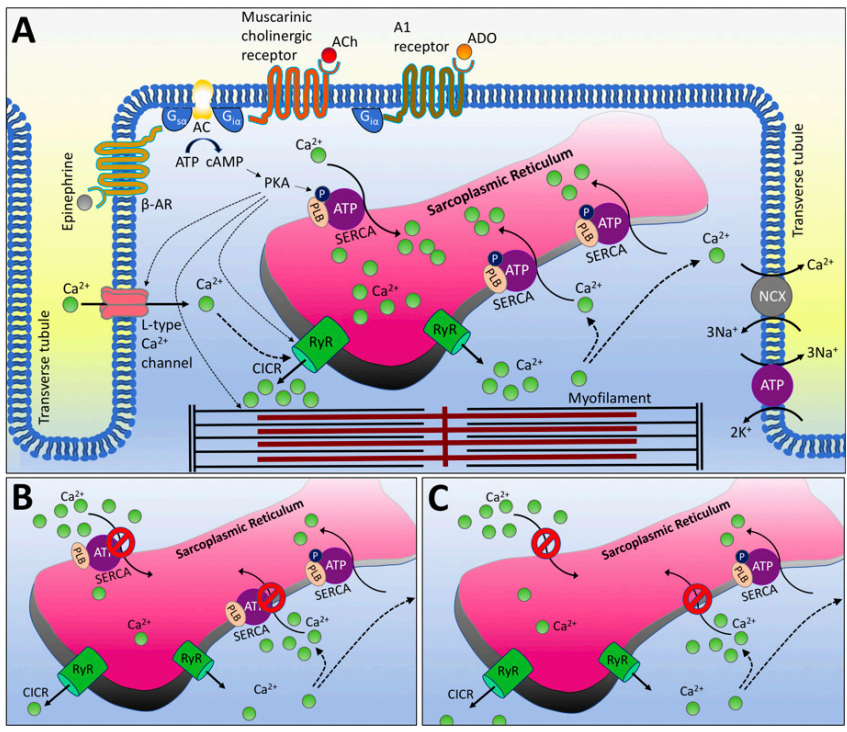

Figure 3 Illustration of a cardiac myocyte featuring a number of calcium-handling proteins (A). Irregular pacing leading to a reduction in phosphorylated phospholamban and subsequent reduction in calcium transport into the sarcoplasmic reticulum (B), and irregular pacing leading to a reduction in SERCA and subsequent reduction in calcium transport into the sarcoplasmic reticulum (C). A1 receptor, adenosine $A 1$ receptor; $A C$, adenylyl cyclase; ACh, acetylcholine; ADO, adenosine; $\beta-A R$, beta adrenergic receptor; cAMP, cyclic AMP; CICR, calcium-induced calcium release; NCX, sodium-calcium exchanger; PKA, protein kinase A; PLB, phospholamban; RyR, ryanodine receptor; SERCA, sarcoplasmic sarcoplasmic/endoplasmic reticulum $\mathrm{Ca}^{2+}$ ATPase.

cardiomyopathy, and who may therefore derive benefit from treatment of the offending arrhythmia with catheter ablation. We believe that these results are worthy of further investigation as they seem to add to our understanding of the factors contributing to LV impairment before and improvement after AF ablation.

There are several limitations in our study. First, it was retrospective and uncontrolled and is thus hypothesis generating rather than definitive. Second, because echocardiograms or cardiac MRI are not routinely obtained after AF ablation, there may be selection bias. Third, echocardiographic or MRI measurements in individual patients were assessed on only two occasions, so we cannot say whether these changes remained consistent in the longer term in their direction or magnitude. Of the patients who qualified for our study based on having a low preablation LVEF, only $\sim 60 \%$ had subsequent echocardiograms to allow for comparison. Fourth, the sample size of patients was relatively small and included only men. Fifth, information about the burden of AF may have provided more insights into our findings. However, improvement in LVEF may indirectly show evidence of the clinical impact of ablation irrespective of knowing the AF burden. Sixth, the use of ACEi/ARB medications was $67 \%$. This was relatively low for a population with impaired LV function, but was unlikely to have affected the main finding of our study. Finally, most patients were evaluated by echocardiography, and some might argue this commonly available tool is not the best way to assess cardiac function, particularly given the constraint of previously reported $\sim 7.5 \%$ interobserver variability in the measurement of 2D-EF. ${ }^{8}$ Furthermore, fibrosis burden as determined by cardiac MRI has been shown to be an important predictor of ablation response in non-ischaemic cardiomyopathy, ${ }^{11}$ and this could provide further insight into our study.

\section{CONCLUSIONS}

In patients with heart failure undergoing AF ablation, both an increase in R-R dispersion on ECG and smaller LVIDd were associated with improvement in LVEF after catheter ablation. Increased R-R dispersion remained significant after adjustment for potential confounders and heart rate. This broadens the existing knowledge on AIC, particularly the notion that irregular electrical activation can cause cardiomyopathy and that the cardiomyopathy may improve with elimination of the irregularity using catheter ablation. These readily available measurements may help to identify those patients most likely to derive LV systolic function improvement after catheter ablation.

\section{Author affiliations}

${ }^{1}$ Department of Cardiovascular Medicine, University of Minnesota Medical School, Minneapolis, Minnesota, USA

'UCLA Medical Center, UCLA Health System, Department of Medicine, University of California, Los Angeles, Los Angeles, California, USA

${ }^{3}$ Cardiovascular Division, University of California, Los Angeles, Los Angeles, California, USA

${ }^{4}$ Cardiovascular Division, University of Minnesota Medical School, Minneapolis Veterans Affairs Health Care System, Minneapolis, Minnesota, USA

${ }^{5}$ Division of Cardiology, University of Minnesota, Minneapolis VA Health Care System, Minneapolis, Minnesota, USA

Contributors Conception and design: RJK, VNT. Administrative support: RJK, VNT. Provision of study materials: RJK, VNT, EB. Collection and assembly of data: RJK, EB, Y-JS, VNT. Data analysis and interpretation: All authors. Manuscript writing: RJK, VNT. Final approval of manuscript: All authors: RJK, VNT, EB, Y-JS.

Funding The authors have not declared a specific grant for this research from any funding agency in the public, commercial or not-for-profit sectors.

Competing interests None declared.

Patient consent for publication Not required.

Provenance and peer review Not commissioned; externally peer reviewed.

Data availability statement Data are available on reasonable request.

Open access This is an open access article distributed in accordance with the Creative Commons Attribution Non Commercial (CC BY-NC 4.0) license, which permits others to distribute, remix, adapt, build upon this work non-commercially, and license their derivative works on different terms, provided the original work is properly cited, appropriate credit is given, any changes made indicated, and the use is non-commercial. See: http://creativecommons.org/licenses/by-nc/4.0/.

\section{REFERENCES}

1. Chen T, Koene R, Benditt DG, et al. Ventricular ectopy in patients with left ventricular dysfunction: should it be treated? J Card Fail 2013;19:40-9.

2. Clark DM, Plumb VJ, Epstein AE, et al. Hemodynamic effects of an irregular sequence of ventricular cycle lengths during atrial fibrillation. J Am Coll Cardiol 1997;30:1039-45. 
3. Anter E, Jessup M, Callans DJ, et al. Atrial fibrillation and heart failure: treatment considerations for a dual epidemic. Circulation 2009;119:2516-25.

4. Jeong Y-H, Choi K-J, Song J-M, et al. Diagnostic approach and treatment strategy in tachycardia-induced cardiomyopathy. Clin Cardiol 2008;31:172-8.

5. Lubitz SA, Benjamin EJ, Ellinor PT. Atrial fibrillation in congestive heart failure. Heart Fail Clin 2010;6:187-200.

6. Naito M, David D, Michelson EL, et al. The hemodynamic consequences of cardiac arrhythmias: evaluation of the relative roles of abnormal atrioventricular sequencing, irregularity of ventricular rhythm and atrial fibrillation in a canine model. Am Heart $J$ 1983;106:284-91.

7. Lang RM, Bierig M, Devereux RB, et al. Recommendations for chamber quantification: a report from the American Society of echocardiography's guidelines and standards Committee and the chamber quantification writing group, developed in conjunction with the European association of echocardiography, a branch of the European Society of cardiology. J Am Soc Echocardiogr 2005;18:1440-63.

8. Shahgaldi K, Gudmundsson P, Manouras A, et al. Visually estimated ejection fraction by two dimensional and triplane echocardiography is closely correlated with quantitative ejection fraction by real-time three dimensional echocardiography. Cardiovasc Ultrasound 2009;7.

9. Ling L-han, Khammy O, Byrne $\mathrm{M}$, et al. Irregular rhythm adversely influences calcium handling in ventricular myocardium: implications for the interaction between heart failure and atrial fibrillation. Circ Heart Fail 2012;5:786-93.

10. Hsu L-F, Jaïs $P$, Sanders $P$, et al. Catheter ablation for atrial fibrillation in congestive heart failure. N Engl J Med 2004;351:2373-83.

11. Prabhu S, Taylor AJ, Costello BT, et al. Catheter ablation versus medical rate control in atrial fibrillation and systolic dysfunction: the CAMERA-MRI study. J Am Coll Cardiol 2017;70:1949-61. 